\title{
Upregulation of long non-coding RNA CCEPR is associated with poor prognosis and contributes to the progression of ovarian cancer through regulating the $\mathrm{Wnt} / \boldsymbol{\beta}$-catenin signaling pathway
}

\author{
ZHEN CHEN, YINGJUN ZHU, XIAODONG FAN, YABO LIU and QINGQING FENG \\ Department of General Gynecology, Tianjin Central Hospital of Gynecology and Obstetrics, \\ Tianjin 300100, P.R. China
}

Received June 29, 2019; Accepted January 16, 2020

DOI: $10.3892 / \mathrm{mmr} .2020 .10979$

\begin{abstract}
Accumulating evidence has demonstrated that the expression of long non-coding RNAs (lncRNAs) is altered in various types of cancer, which may prove beneficial for their use as biomarkers. Cervical carcinoma expressed PCNA regulatory lncRNA (CCEPR) is a recently identified lncRNA, which has an important role in regulating cell proliferation and apoptosis in cervical and bladder cancer; however, whether CCEPR is involved in the progression of ovarian cancer (OC) remains largely unclear. The aim of the present study was to determine the clinical significance of CCEPR in OC and to investigate its biological roles. Cell Counting Kit- 8 assay was used to analyze cell proliferation, Transwell assay was used to assess invasion, flow cytometric analysis was used to analyze apoptosis, and western blotting was used to perform mechanistic studies. CCEPR expression levels were significantly elevated in OC tissues compared with adjacent non-cancer tissues. Similarly, significant increases in CCEPR expression were observed in OC cell lines (SK-OV-3 and OVCAR-3) compared with the ovarian surface epithelial cell line, HOSEpiC. The increased expression levels of CCEPR were associated with increased invasion, higher International Federation of Gynecology and Obstetrics stage and a poorer overall survival rate. In vitro, the genetic silencing of CCEPR decreased the cell proliferation rate and invasive ability of OC cells, and promoted apoptosis. CCEPR-silenced OC cells also demonstrated decreased expression levels of four proteins involved in the $\mathrm{Wnt} / \beta$-catenin signaling pathway: Cyclin D1, $\beta$-catenin, Myc and matrix metallopeptidase-7. In conclusion, the present study demonstrated that increased expression levels
\end{abstract}

Correspondence to: Professor Yingjun Zhu, Department of General Gynecology, Tianjin Central Hospital of Gynecology and Obstetrics, 156 Nankai Sanma Road, Nankai, Tianjin 300100, P.R. China

E-mail: zhuyingjun66@yeah.net

Key words: cervical carcinoma expressed PCNA regulatory lncRNA, ovarian cancer, progression, Wnt/ $\beta$-catenin of CCEPR may predict poor prognosis in patients with OC and contribute to the progression of $\mathrm{OC}$ through regulating the Wnt/ $\beta$-catenin signaling pathway.

\section{Introduction}

Ovarian cancer (OC) is the most lethal form of gynecological malignancy and the fifth most common cause of cancer-associated mortality among women worldwide $(1,2)$. The etiology of OC remains unclear and may be related to the following factors: Genetics, early menarche, ovulation abnormalities, nulliparity or not breastfeeding. Despite improvements in the diagnosis, surgery and chemotherapy treatments available for OC over the past few decades, the overall survival (OS) of patients with OC has not changed, with a 5-year survival rate for all stages of $35-38 \%(3,4)$. One of the most prominent factors contributing to this poor outcome is the fact that the majority of patients with OC are diagnosed at a late stage (stage III-IV), which is closely associated with tumor recurrence and metastasis (5). A number of previous studies have reported that the initiation and development of OC is a multi-step pathological process involving a variety of alterations in gene expression and gene variants $(6,7)$. Therefore, an enhanced knowledge of the mechanism, and the identification of novel prognostic and therapeutic targets is crucial to devising novel, effective therapies for patients with $\mathrm{OC}$.

Long non-coding RNAs (lncRNAs) are a group of non-coding RNAs $>200$ nucleotides in length that do not encode proteins (8). The majority of lncRNAs are evolutionarily conserved and serve important functions in the modulation of gene expression at the post-transcriptional level $(9,10)$; previous studies have observed that lncRNAs are involved in regulating various cellular processes, such as cell proliferation, cell cycle, cell apoptosis, epithelial-mesenchymal transition, metastasis and chemosensitivity $(11,12)$. To date, accumulating evidence has suggested that the aberrant expression of lncRNAs is related to the initiation and development of various types of human malignancy, and that lncRNAs may be considered potential candidate prognostic biomarkers (13). For example, Liu et al (14) demonstrated that long intergenic non-protein coding RNA 460 served a role in OC progression and suggested that it could be used as a novel therapeutic 
strategy for OC. Li et al (15) observed that the upregulation of SPRY4 intronic transcript 1 accelerated tumorigenesis in patients with OC and its upregulation was associated with a poor prognosis. In addition, Luo and Liu (16) reported that TTN antisense RNA 1 (TTN-AS1) may have a carcinogenic role in lung adenocarcinoma through destabilizing the PTEN gene to activate the PI3K/AKT signaling pathway, thus suggesting that TTN-AS1 may be a potential biomarker for lung adenocarcinoma treatment. However, the lncRNA-mediated regulatory mechanism driving the initiation and development of OC remains largely unknown.

Cervical carcinoma expressed PCNA regulatory lncRNA (CCEPR) has been reported to serve an oncogenic role in cervical cancer and urothelial bladder carcinoma $(17,18)$; however, the clinical significance and biological function of CCEPR in OC has not yet been illustrated. In the present study, the oncogenic function of CCEPR in OC and its prognostic value in patients with OC was confirmed. CCEPR knockdown suppressed the progression of OC by decreasing cell proliferation and invasion, and inducing cell apoptosis. In addition, CCEPR inhibition was observed to decrease the expression levels of proteins involved in the Wnt $/ \beta$-catenin signaling pathway, including cyclin D1, $\beta$-catenin, Myc and matrix metallopeptidase-7 (MMP-7). In conclusion, the present study provided a novel insight into the biological role and regulatory pathways of CCEPR in OC progression, and identified CCEPR as a promising prognostic and therapeutic biomarker for OC intervention.

\section{Materials and methods}

Patient studies. The present study was conducted in accordance with the Declaration of Helsinki 1991 and was approved by the Institutional Review Board of Tianjin Central Hospital of Gynecology and Obstetrics (Tianjin, China). Written informed consent was obtained from all patients. A total of 70 paired OC tissues and corresponding normal tissues were collected from patients (aged 28-72 years) at Tianjin Central Hospital of Gynecology and Obstetrics between February 2007 and August 2015. The following inclusion criteria were required: i) Good indication of suitability for surgical intervention; ii) must not have received radiotherapy or chemotherapy prior to surgery; and iii) complete clinical history. The following exclusion criteria were required: i) Previous surgical intervention; ii) severe liver and/or kidney dysfunction; iii) abnormal coagulation; and iv) incomplete clinical history. All clinical and pathological information was obtained from each patient's history record. An experienced gynecological pathologist at the hospital assessed all tissue specimens. After surgery, each patient was followed up every 3 months for a period of 60 months. The OS time was defined as the time between the date of surgery and the date of death or last follow-up. All clinical specimens were immediately snap-frozen in liquid nitrogen and subsequently stored at $-80^{\circ} \mathrm{C}$ until required for RNA extraction.

Cell lines and reagents. The two human OC cell lines, SK-OV-3 (cat. no. HTB-77) and OVCAR-3 (cat. no. HTB-161), were purchased from the American Type Culture Collection. The human ovarian surface epithelial cell line HOSEpiC (cat. no. 7310) was obtained from ScienCell Research Laboratories, Inc. All cells were cultured in RPMI-1640 medium (HyClone; GE Healthcare Life Sciences), supplemented with 10\% FBS (HyClone; GE Healthcare Life Sciences) and $100 \mu \mathrm{g} / \mathrm{ml}$ penicillin/streptomycin. All cells were cultured at $37^{\circ} \mathrm{C}$ in a humidified atmosphere containing $5 \% \mathrm{CO}_{2}$.

Cell transfection. Two short hairpin (sh)RNAs targeting CCEPR, shCCERP1 (cat. no. H-4861, 5'-ATGTTATAGCTA AATGGATGTGACTAG-3') and shCCEPR2 (cat. no. H-4862, 5'-CATTTTATGTCTTGACAATGCCTCGATTTG-3'), and the corresponding scrambled shRNAs [negative control (NC)], shNC1 (cat. no. H-4861-1, 5'-ACTGGCTCATTACCTGAG GAAATGTGT-3') and shNC2 (cat. no. H-4862-1, 5'-AAT GTTGGACCCTTTACAGTTGGAAATC-3'), were designed and cloned into psi-H1 vector from GeneCopoeia, Inc (Guangzhou, China). Sequences of all oligonucleotides were confirmed by Sanger sequencing (Thermo Fisher Scientific, Inc.). Upon reaching $80 \%$ confluence, cells in 6-well plates at $8 \times 10^{6}$ cells/well were transfected with $2 \mu \mathrm{g} / \mathrm{well}$ shRNA or NC using Lipofectamine ${ }^{\circledR} 2000$ reagent (Invitrogen; Thermo Fisher Scientific, Inc.) for $6 \mathrm{~h}$ at $37^{\circ} \mathrm{C}$, according to the manufacturer's protocol. Cells were incubated with RPMI-1640 at $37^{\circ} \mathrm{C}$ supplemented with $10 \%$ FBS for $48 \mathrm{~h}$ prior to subsequent experimentation. The efficiency of cell transfection was analyzed using reverse transcription-quantitative PCR (RT-qPCR) analysis.

Cell proliferation assays. To measure the cell proliferation rate, a total of $3 \times 10^{3}$ transfected OC cells/well were plated into 96-well plates and cultured overnight. Subsequently, $10 \mu 1$ Cell Counting Kit-8 (CCK-8) solution (Dojindo Molecular Technologies, Inc.) was added to each well at the at $0,24,48$ and $72 \mathrm{~h}$. Following incubation for $3 \mathrm{~h}$ at $37^{\circ} \mathrm{C}$, the absorbance was measured at $450 \mathrm{~nm}$ using an automatic microplate reader (Bio-Rad Laboratories, Inc.).

Western blotting. Total protein was extracted from cells with ice-cold mammalian cell total protein lysis buffer (Sangon Biotech Co., Ltd.) and centrifuged at 13,000 x g for $30 \mathrm{~min}$ to obtain the protein samples at $4^{\circ} \mathrm{C}$. Total protein was quantified using a bicinchoninic acid assay kit (Pierce; Thermo Fisher Scientific, Inc.) and $25 \mu \mathrm{g}$ protein/lane was separated via SDS-PAGE on a $12 \%$ gel. The separated proteins were subsequently transferred onto PVDF membranes (EMD Millipore) and blocked with 5\% non-fat milk in TBS- $0.1 \%$ Tween 20 for $1 \mathrm{~h}$ at $37^{\circ} \mathrm{C}$. The membranes were incubated with the following primary antibodies overnight at $4^{\circ} \mathrm{C}$ : Anti-Myc $(1: 1,000$; cat. no. D199941; Sangon Biotech Co., Ltd.), anti-MMP-7 (1:2,000; cat. no. MAB9071-100; R\&D Systems, Inc.), anti-cyclin D1 (1:1,000; cat. no. D198702; Sangon Biotech Co., Ltd.), anti- $\beta$-catenin (1:500; cat. no. D199519; Sangon Biotech Co., Ltd.) and anti-GAPDH (1:2,500; cat. no. D190090; Sangon Biotech Co., Ltd.). Following the primary antibody incubation, membranes were incubated with horseradish peroxidase (HRP)-conjugated secondary antibodies $(1: 2,000$; cat. no. D110098; Sangon Biotech Co., Ltd.) for $1 \mathrm{~h}$ at $37^{\circ} \mathrm{C}$. Protein bands were visualized using an enhanced chemiluminescence kit (Thermo Fisher Scientific, Inc.) and protein expression was semi-quantified using a v4.6.6 Quantity One 
software (Bio-Rad Laboratories, Inc.), with GAPDH as the loading control.

Bioinformatics analysis. The Kyoto Encyclopedia of Genes and Genomes (KEGG) pathway online database (https://www. genome.jp/kegg/pathway.html) was used to predict the downstream signaling pathways of CCEPR.

ELISA. Human Bcl-2 (cat. no. CSB-E08853h), Bax (cat. no. CSB-E09344h) and caspase-3 (cat. no. CSB-E08856h) ELISA kits (Cusabio Technology LLC) were used to analyze the expression levels of Bcl-2, Bax and caspase-3 in transfected cells. Briefly, a total of $1 \times 10^{6}$ transfected OC cells were harvested and lysed with lysis buffer (Cusabio Technology LLC) provided by the kits on ice for $30 \mathrm{~min}$ at $4^{\circ} \mathrm{C}$ and centrifuged at $13,000 \mathrm{x} \mathrm{g}$ for $30 \mathrm{~min}$ to obtain the supernatants at $4^{\circ} \mathrm{C}$. A total of $100 \mu \mathrm{l} /$ well supernatant was added to the 96-well ELISA plates and incubated at $37^{\circ} \mathrm{C}$ for $30 \mathrm{~min}$. Subsequently, $100 \mu 1 \mathrm{XX}$ HRP-conjugate solution was added to each well and incubated for $30 \mathrm{~min}$ at $37^{\circ} \mathrm{C}$. Then, $90 \mu \mathrm{l}$ tetramethylbenzidine substrate solution/well was added to the plates and incubated for $20 \mathrm{~min}$ at $37^{\circ} \mathrm{C}$ in the dark. Finally, $50 \mu \mathrm{l}$ stop solution was added to each well to terminate the reaction and the optical density value at $570 \mathrm{~nm}$ was measured using an automatic microplate reader (Bio-Rad Laboratories, Inc.).

$R T-q P C R$. Total RNA was extracted from OC cell lines and cancer/normal tissues using TRIzol ${ }^{\circledR}$ reagent (Invitrogen; Thermo Fisher Scientific, Inc.), according to the manufacturer's protocol. The quality was assessed using a SmartSpec Plus spectrophotometer (Bio-Rad Laboratories, Inc.) and $1 \mu \mathrm{g}$ RNA was reverse transcribed into cDNA using a cDNA Synthesis kit (Toyobo Life Science), according to the manufacturer's protocol. qPCR was subsequently performed using the $\mathrm{SYBR}^{\circledast}$ qPCR mix (Toyobo Life Science) and a 7900HT Sequence Detection system (Applied Biosystems; Thermo Fisher Scientific, Inc.), according to the manufacturer's protocol. The following primer pairs (Sangon Biotech Co., Ltd.) were used for qPCR: CCEPR, forward, 5'-AAGGTCCCA GGATACTCGC-3', reverse, 5'-GTGTCGTGGACTGGCAAA AT-3'; and GAPDH, forward, 5'-CGCTCTCTGCTCCTCCTG TTC-3' and reverse, 5'-ATCCGTTGACTCCGACCTTCAC-3'. The following thermocycling conditions were used for qPCR: Initial denaturation at $95^{\circ} \mathrm{C}$ for $60 \mathrm{sec}$; followed by 40 cycles at $95^{\circ} \mathrm{C}$ for $10 \mathrm{sec}, 60^{\circ} \mathrm{C}$ for $30 \mathrm{sec}$ and $72^{\circ} \mathrm{C}$ for $45 \mathrm{sec}$, finally at $4^{\circ} \mathrm{C}$ for $30 \mathrm{~min}$. Expression levels were quantified using the $2^{-\triangle \Delta C q}$ method (19) and normalized to the internal reference gene GAPDH.

Flow cytometry. A total of $7 \times 10^{4}$ transfected cells/well were plated into 6-well plates and cultured for $48 \mathrm{~h}$ in $5 \% \mathrm{CO}_{2}$, prior to being harvested. Subsequently, cells were centrifuged at $1,000 \mathrm{x} \mathrm{g}$ for $10 \mathrm{~min}$ at $37^{\circ} \mathrm{C}$ and $1 \times 10^{6}$ cells were fixed overnight with $70 \%$ ice-cold ethanol at $4^{\circ} \mathrm{C}$. The cells were resuspended in PBS containing $50 \mathrm{mg} / \mathrm{ml}$ RNase A (cat. no. ST576; Beyotime Institute of Biotechnology) for $30 \mathrm{~min}$ at $37^{\circ} \mathrm{C}$, and blocked with $10 \%$ goat serum (Solarbio Science \& Technology Co., Ltd., Beijing, China) for $2 \mathrm{~h}$ at $37^{\circ} \mathrm{C}$. Next, the cells were stained with $3 \mu \mathrm{l}$ propidium iodide and
$5 \mu \mathrm{l}$ Annexin V-FITC (Thermo Fisher Scientific, Inc.) in the dark for $1 \mathrm{~h}$ at $37^{\circ} \mathrm{C}$. The apoptotic cells were visualized using a BD FACSCalibur ${ }^{\mathrm{TM}}$ flow cytometer (BD Biosciences) and subsequently analyzed using BD CellQuest ${ }^{\mathrm{TM}}$ Pro version 5.1 software (BD Biosciences).

Transwell invasion assay. The invasion assay was performed using a 24-well Boyden chamber (pore size, $8 \mu \mathrm{m}$; Corning, Inc.). A total of $5 \times 10^{4}$ transfected cells/well were resuspended in $200 \mu 1$ serum-free RPMI-1640 medium and were plated in the upper chambers, which were precoated with Matrigel. A total of $500 \mu 1$ RPMI-1640 medium supplemented with $10 \%$ FBS was plated in the lower chambers as a chemoattractant. After $48 \mathrm{~h}$, the invasive cells were fixed with $4 \%$ paraformaldehyde (Sigma-Aldrich; Merck KGaA) at $37^{\circ} \mathrm{C}$ for $30 \mathrm{~min}$ and subsequently stained with $0.5 \%$ crystal violet (Sigma-Aldrich; Merck KGaA) at $37^{\circ} \mathrm{C}$ for $15 \mathrm{~min}$. Stained cells were counted in five randomly selected fields using an Olympus light microscope at x200 magnification (Olympus Corporation).

Statistical analysis. Statistical analysis was performed using GraphPad Prism 5.0 software (GraphPad Software, Inc.). Data are presented as the mean \pm SD and are representative of $\geq 3$ experimental repeats. The association between CCEPR expression and clinicopathological variables was analyzed using a $\chi^{2}$ test; Kaplan-Meier curves and the log-rank test were used to analyze the association between CCEPR expression levels and OS in patients with OC; Student's t-tests were used to identify statistical differences between two groups; and differences among $>2$ groups were analyzed using one-way ANOVA followed by a Bonferroni's correction post hoc test. $\mathrm{P}<0.05$ was considered to indicate a statistically significant difference.

\section{Results}

CCEPR is highly expressed in OC tissues and cell lines. To determine the potential role of CCEPR in the progression of OC, RT-qPCR was performed to analyze the expression levels of CCEPR in 70 OC and matched normal tissues. The expression levels of CCEPR were significantly increased in OC tissues compared with the matched normal tissues (Fig. 1A; $\mathrm{P}<0.05)$. Furthermore, CCEPR expression was also examined in two OC cell lines, SK-OV-3 and OVCAR-3. Compared with the HOSEpiC cell line, CCEPR expression levels were significantly increased in the two OC cell lines (Fig. 1B and C; $\mathrm{P}<0.05)$.

CCEPR expression is associated with the clinicopathological features and prognosis of patients with $O C$. To determine the clinical value of CCEPR in OC, the relationship between the expression levels of CCEPR and clinicopathological variables was analyzed. Based on the median expression levels of CCEPR in OC tissues, patients with OC were divided into two groups: High expression group $(n=35)$ and low expression group $(\mathrm{n}=35)$. Increased CCEPR expression levels were associated with an increased invasive ability and a higher International Federation of Gynecology and Obstetrics (FIGO) stage (Table I; $\mathrm{P}<0.05$ ), but not with patients' age, pathological type, differentiation, tumor size, CA125 levels or lymph node 
A

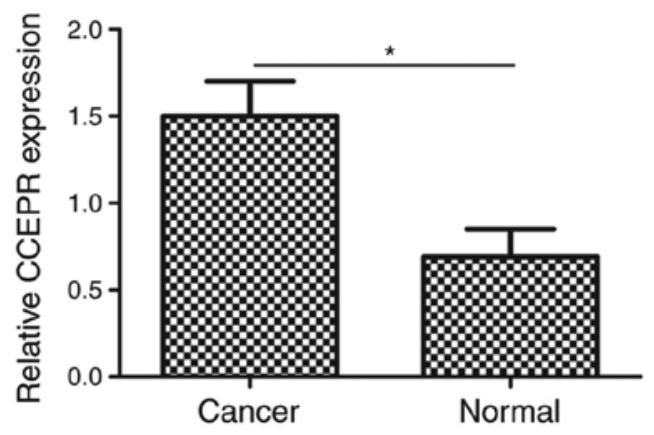

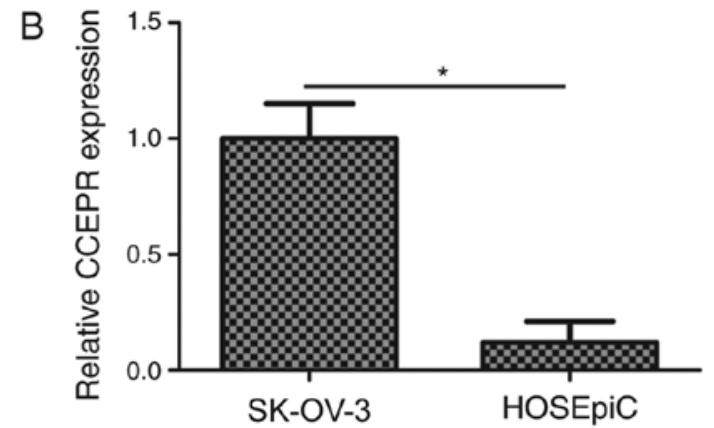

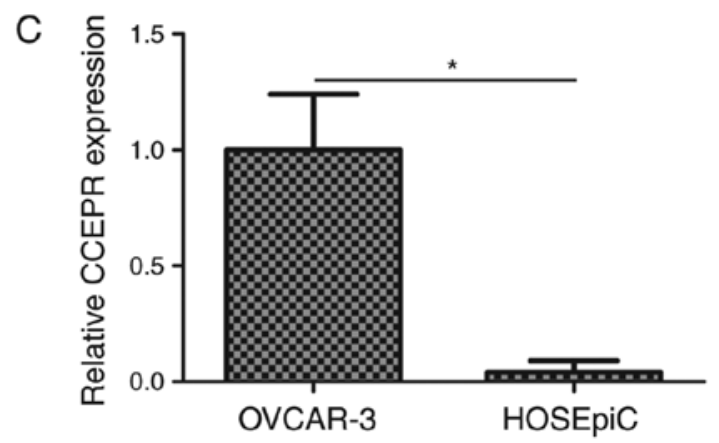

Figure 1. CCEPR expression levels are increased in human OC tissues and cell lines. Reverse transcription-quantitative PCR was used to analyze CCEPR expression levels in (A) 70 paired OC tissues and corresponding normal tissues, (B) the OC cell line SK-OV-3 and HOSEpiC cells, and (C) the OC cell line OVCAR-3 and HOSEpiC cells. Data are presented as the mean \pm standard deviation of $\geq 3$ individual experiments. " $\mathrm{P}<0.05$. CCEPR, cervical carcinoma expressed PCNA regulatory lncRNA; OC, ovarian cancer.

metastasis. Kaplan-Meier curves revealed that the 5-year OS rate was significantly lower in patients within the high expression group (11.43\%) compared with patients in the low expression group (25.71\%; Fig. $2 \mathrm{~A} ; \mathrm{P}<0.05)$. The median survival time of patients with $\mathrm{OC}$ in the low expression group was 45.0 months, whereas the median survival time of patients with OC in the high expression group was 19.6 months. These data suggested that increased CCEPR expression levels may be related to aggressive clinicopathological behaviors and may predict an unfavorable prognosis in patients with OC.

CCEPR knockdown suppresses the proliferation and invasion of OC cells. Given the association of CCEPR expression with invasion and FIGO stage in OC, the biological effects of CCEPR on the proliferation rate and invasive ability of OC cells were further investigated by loss-off function assays. CCEPR expression levels were significantly decreased in SK-OV-3 and OVCAR-3 cells following transfection with shCCEPR 1 compared with shNC1-transfected cells (Fig. 2B; P<0.05); however, transfection with shCCEPR 2 only significantly decreased expression levels in OVCAR-3 cells compared with shNC2-transfected cells (Fig. 2C). Therefore, the shCCEPR1 shRNA was chosen for subsequent experiments. The CCK- 8 assay revealed that the proliferative ability of cells was markedly suppressed in shCCEPR-1-transfected SK-OV-3 and OVCAR-3 cells compared with the shNC1-transfected group (Fig. 2D and E; $\mathrm{P}<0.05$ ). In addition, a Transwell invasion assay was performed to investigate the effect of CCEPR expression on tumor cell invasion. CCEPR knockdown significantly decreased the invasive abilities of SK-OV-3 and OVCAR-3 cells (Fig. 3A-D; P<0.05) compared with the cells transfected with the shNC1. These findings suggested that the knockdown of CCEPR may impede cell growth and invasion in vitro.

CCEPR knockdown induces apoptosis of OC cells. To further confirm whether CCEPR knockdown suppressed OC cell proliferation through regulating cell apoptosis, flow cytometric analysis was performed. The results revealed that the apoptotic rate in the shCCEPR1-transfected cells was significantly increased compared with the shNC1-transfected cells (Fig. 4A-D; P<0.05). Furthermore, the expression levels of cell apoptosis-related proteins, including Bcl-2, Bax and caspase-3 were analyzed using ELISA following CCEPR knockdown. The protein expression levels of $\mathrm{Bcl}-2$ were significantly decreased in OC cells post-transfection with shCCEPR1 compared with the shNC1-transfected cells (Fig. 4E; P<0.05), whereas the protein expression levels of Bax and caspase- 3 were significantly increased in shCCEPR1-transfected cells compared with shNC1-transfected cells (Fig. 4F and G; $\mathrm{P}<0.05)$. These results suggested that the knockdown of CCEPR may induce apoptosis of OC cells in vitro.

Wnt/ $\beta$-catenin signaling pathway is associated with the role of CCEPR in OC cells. The potential mechanism through which CCEPR regulates OC progression was subsequently investigated. KEGG analysis was used to predict the downstream signaling pathways of CCEPR and the Wnt//-catenin signaling pathway was highly enriched; thus, western blotting was used to verify this. Significantly decreased expression levels of proteins involved in the Wnt/ $\beta$-catenin signaling pathway, including cyclin D1, $\beta$-catenin, Myc and MMP-7, were observed in shCCEPR1-transfected OC cells compared with the shNC1-transfected cells (Fig. 5A-D; P<0.05). These 
Table I. Association between CCEPR expression levels and clinicopathological variables in patients with ovarian cancer.

\begin{tabular}{|c|c|c|c|c|c|}
\hline \multirow[b]{2}{*}{ Variable } & \multirow[b]{2}{*}{$\mathrm{N}$} & \multicolumn{2}{|c|}{ CCEPR expression } & \multirow[b]{2}{*}{$\chi^{2}$} & \multirow[b]{2}{*}{ P-value } \\
\hline & & Low & High & & \\
\hline Age (years) & & & & 2.809 & 0.094 \\
\hline$<50$ & 37 & 15 & 22 & & \\
\hline$\geq 50$ & 33 & 20 & 13 & & \\
\hline Differentiation & & & & 2.885 & 0.089 \\
\hline Well & 29 & 18 & 11 & & \\
\hline Poor & 41 & 17 & 24 & & \\
\hline Tumor size $(\mathrm{cm})$ & & & & 2.692 & 0.101 \\
\hline$<4$ & 52 & 29 & 23 & & \\
\hline$\geq 4$ & 18 & 6 & 12 & & \\
\hline CA125 levels (U/ml) & & & & 3.049 & 0.081 \\
\hline$<5,000$ & 25 & 16 & 9 & & \\
\hline$\geq 5,000$ & 45 & 19 & 26 & & \\
\hline Pathological type & & & & 0.402 & 0.526 \\
\hline Serous & 58 & 30 & 28 & & \\
\hline Mucinous & 12 & 5 & 7 & & \\
\hline Invasion & & & & 4.242 & 0.039 \\
\hline $\mathrm{T} 1+\mathrm{T} 2$ & 22 & 15 & 7 & & \\
\hline $\mathrm{T} 3+\mathrm{T} 4$ & 48 & 20 & 28 & & \\
\hline Lymph node metastasis & & & & 1.296 & 0.255 \\
\hline Absent (N0) & 16 & 10 & 6 & & \\
\hline Present (N1-N3) & 54 & 25 & 29 & & \\
\hline $\begin{array}{l}\text { International federation of } \\
\text { gynecology and obstetrics stage }\end{array}$ & & & & 17.425 & $<0.001$ \\
\hline I-II & 27 & 22 & 5 & & \\
\hline III-IV & 43 & 13 & 30 & & \\
\hline
\end{tabular}

CCEPR, cervical carcinoma expressed PCNA regulatory lncRNA.

results suggested that CCEPR may be involved in the progression of OC through regulating the $\mathrm{Wnt} / \beta$-catenin signaling pathway.

\section{Discussion}

Accumulating evidence has demonstrated that lncRNAs are frequently dysregulated in OC and serve as important regulators of numerous cellular processes $(20,21)$. For example, TP73-AS1 was highly expressed in OC tissues and cells, whereas the knockdown of TP73-AS1 expression significantly suppressed proliferation, and the migratory and invasive ability of SK-OV-3 cells (22). Growth arrest specific 5 has been reported to serve a tumor suppressive role towards the proliferation of OC cells through suppressing microRNA (miR)-21 expression and increasing sprouty RTK signaling antagonist 2 expression (23). Nuclear paraspeckle assembly transcript 1 promoted OC cell metastasis via regulating the miR-382-3p/ROCK1 signaling pathway (24); and lncRNA SRY-box transcription factor 4 has been reported to exert an oncogenic effect on the development of OC by increasing cell proliferation and reducing cell apoptosis (25). These findings indicated that lncRNAs may demonstrate potential as novel biomarkers and therapeutic targets for the treatment of OC. In the present study, increased CCEPR expression levels were associated with a poor prognosis in patients with $\mathrm{OC}$, and contributed to the progression of $\mathrm{OC}$ through regulating the $\mathrm{Wnt} / \beta$-catenin signaling pathway.

CCEPR is a newly identified lncRNA 2,502 nucleotides in length, which is localized on the human chromosome 10q21.1 region (26). A previous study demonstrated that increased CCEPR expression was significantly correlated with a higher TNM stage and histological grade in urothelial bladder carcinoma, in addition to predicting a poorer prognosis (17). Consistent with these findings, in the present study it was demonstrated that the expression levels of CCEPR were significantly increased in OC tissues and cell lines. Furthermore, higher CCEPR expression levels were significantly associated with poor OS and unfavorable clinicopathological behaviors, including an increased invasive ability and an advanced FIGO stage. Notably, the present results revealed that the 5 -year OS rate was significantly lower in patients with OS 
A

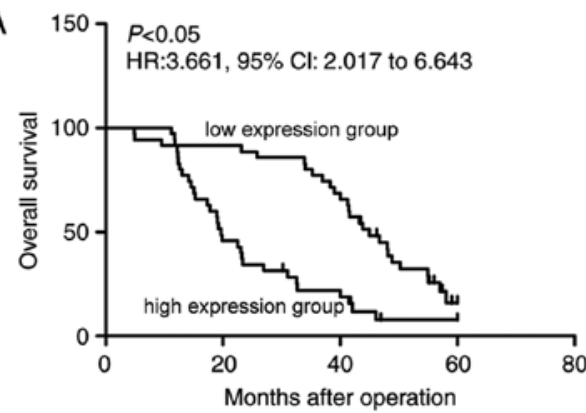

C

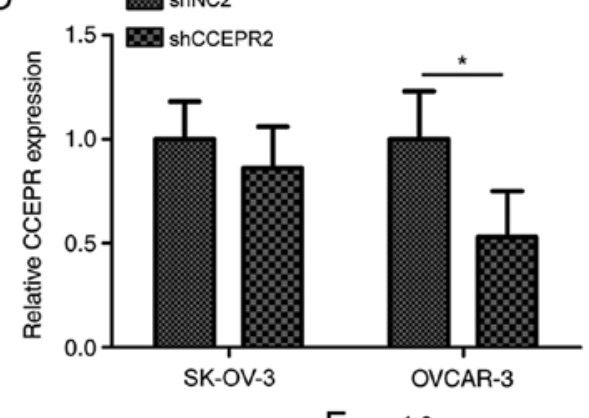

B $\quad$ shNC1

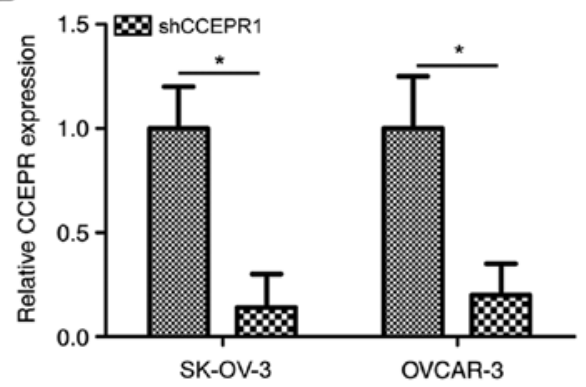

D

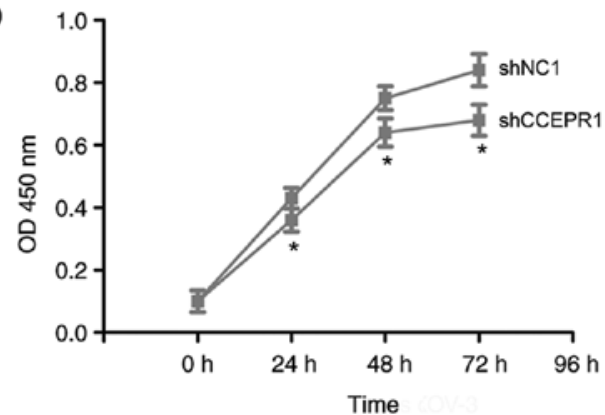

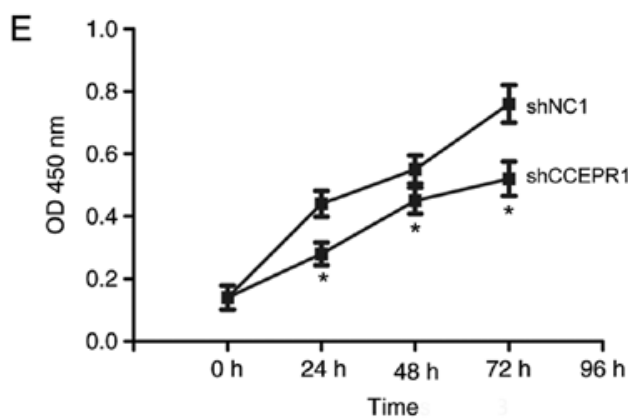

Figure 2. Effects of CCEPR knockdown on the proliferation of OC cells. (A) Patients with OC in the high CCEPR expression group ( $\mathrm{n}=35$ ) demonstrated poorer overall survival compared with patients in the low CCEPR expression group $(n=35)$. Expression levels of CCEPR were detected by reverse transcription-quantitative PCR in SK-OV-3 and OVCAR-3 cells transfected with specific shRNAs (B) shCCEPR1 and shNC1, and (C) shCCEPR2 and shNC2 Cell Counting Kit-8 assays were used to detect the effects of shCCEPR1 on the proliferative ability of (D) SK-OV-3 and (E) OVCAR-3 cells compared with the shNC1. Data are presented as the mean \pm SD of $\geq 3$ individual experiments. ${ }^{*}<0.05$. CCEPR, cervical carcinoma expressed PCNA regulatory lncRNA; $\mathrm{NC}$, negative control; OC, ovarian cancer; OD, optical density; sh/shRNA, short hairpin RNA.

A
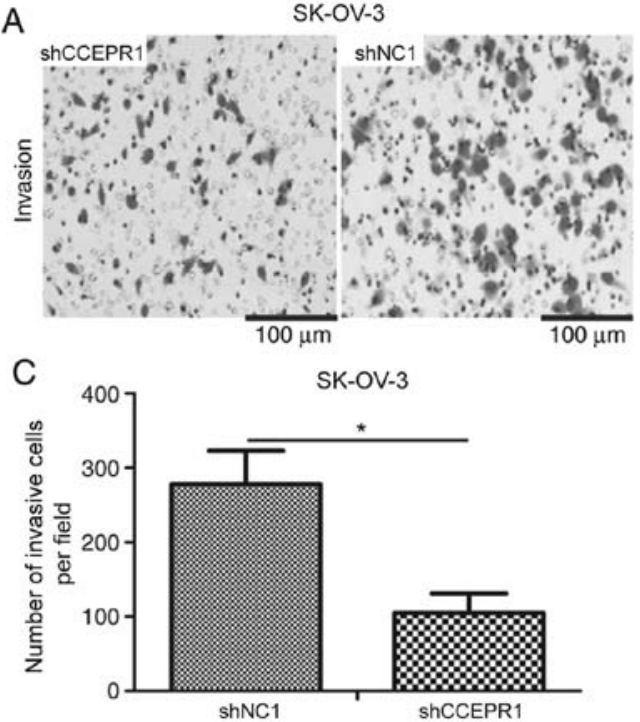

B
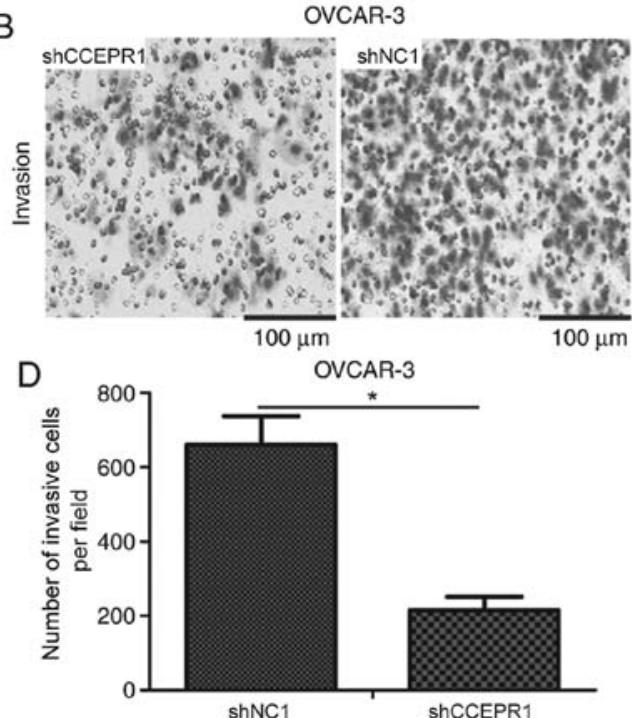

Figure 3. Effects of CCEPR knockdown on the invasive ability of ovarian cancer cells. Transwell invasion assays were used to analyze the invasive ability of shCCEPR1-transfected (A) SK-OV-3 and (B) OVCAR-3 cells compared with shNC1-transfected cells. Scale bars, $100 \mu$ m. (B) CCEPR knockdown significantly impaired the invasive ability of (C) SK-OV-3 and (D) OVCAR-3 cells compared with the cells transfected with shNC1. Data are presented as the mean \pm SD of $\geq 3$ individual experiments. "P $<0.05$. CCEPR, cervical carcinoma expressed PCNA regulatory lncRNA; NC, negative control; sh, short hairpin RNA. 
A

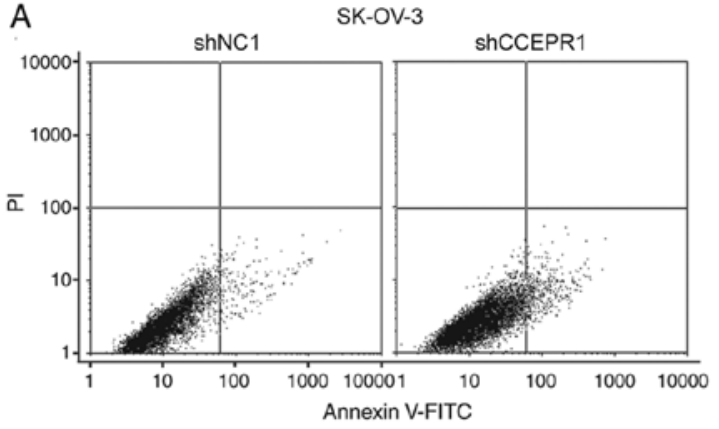

C

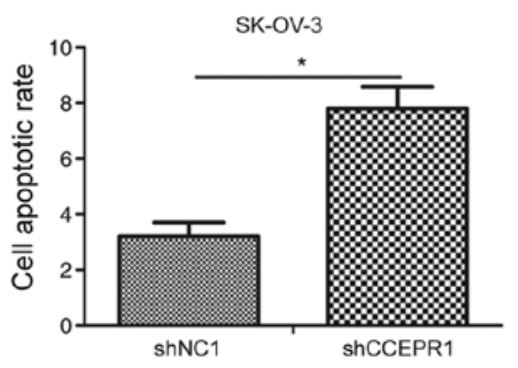

B
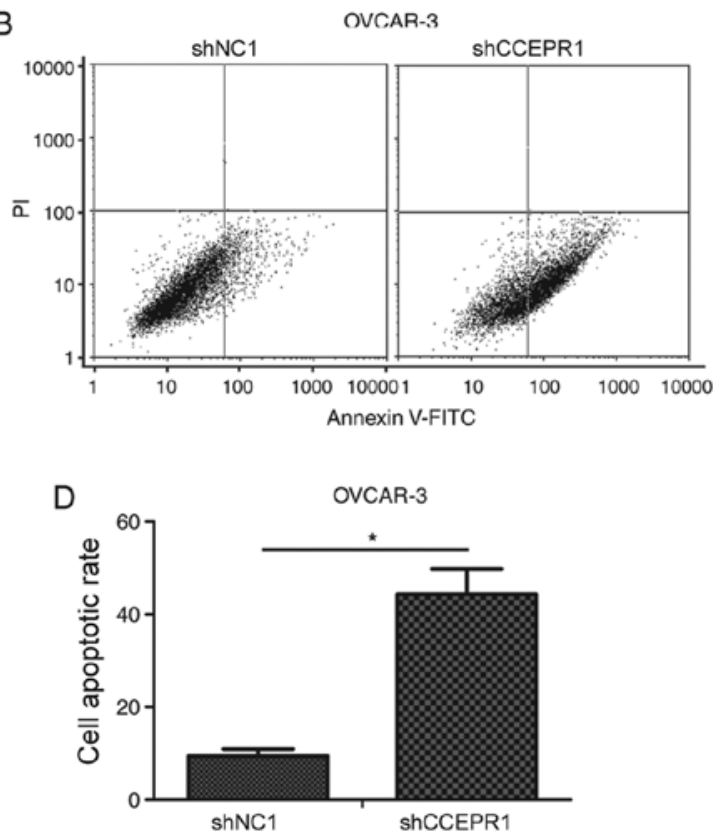

E

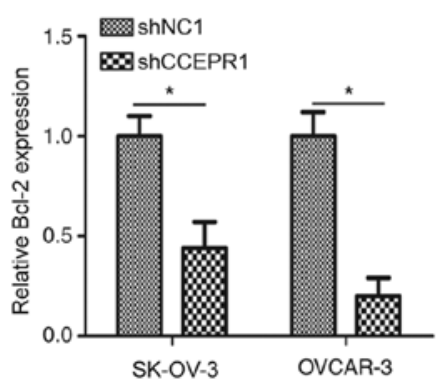

$\mathrm{F}$

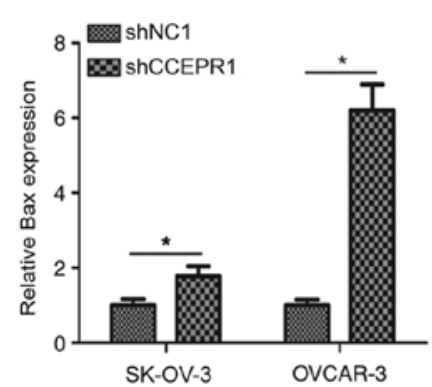

G

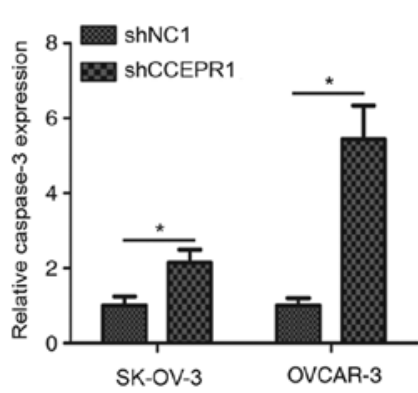

Figure 4. CCEPR knockdown induces the apoptosis of ovarian cancer cells. Apoptotic rate of shCCEPR1-transfected (A) SK-OV-3 and (B) OVCAR-3 cells compared with shNC1-transfected cells, as assessed using flow cytometric analysis. CCEPR knockdown significantly increased the apoptotic rate of (C) SK-OV-3 and (D) OVCAR-3 cells compared with cells transfected with shNC1. Apoptosis-related markers, (E) Bcl-2, (F) Bax and (G) caspase-3 were detected using ELISA in SK-OV-3 and OVCAR-3 cells transfected with shCCEPR1 or shNC1. Data are presented as the mean \pm SD of $\geq 3$ individual experiments. "P<0.05. CCEPR, cervical carcinoma expressed PCNA regulatory lncRNA; NC, negative control; sh, short hairpin RNA.

that demonstrated high expression levels of CCEPR compared with those patients with low expression levels of CCEPR. These results suggested that increased CCEPR expression may be used as a promising prognostic biomarker for patients with OC.

There is increasing interest regarding the roles of CCEPR in the proliferation and apoptosis of human cancer $(17,18)$. Thus, to evaluate the function of CCEPR, SK-OV-3 and OVCAR-3 cells were used to detect the effect of CCEPR knockdown on cell behaviors in vitro. The results indicated that CCEPR expression levels were significantly decreased in SK-OV-3 and OVCAR-3 cells following transfection with shCCEPR1. Using the CCK-8 assay, Transwell invasion assay and flow cytometric analysis, it was observed that transfection of OC cell lines with shCCEPR1 significantly suppressed proliferation and invasion, whilst promoting cell apoptosis. On account of these observations, it was hypothesized that CCEPR may serve an oncogenic role in OC cells. These data were consistent with previous studies, in which CCEPR was demonstrated to be an oncogene in cervical cancer and urothelial bladder carcinoma $(17,18)$.

The Wnt/ $\beta$-catenin signaling transduction pathway regulates development and homeostasis, but is also tightly associated with the development of cancer $(27,28)$. Activation of the Wnt/ $\beta$-catenin signaling pathway has been demonstrated to mediate the initiation and progression of OC through regulating cell proliferation, apoptosis and metastasis (29). In addition, the Wnt/ $\beta$-catenin signaling pathway has been reported to be an important modulator of cell invasion, and it has been observed to participate in cisplatin-induced chemoresistance of OC (30). In the Wnt/ $\beta$-catenin signaling pathway, the $\beta$-catenin and T-cell factor complex translocates to the nucleus, where it drives the transcription of downstream genes, such as MYC, cyclin D1 and MMP-7, which promotes the transformation of a normal cell into a tumor cell $(31,32)$. Using KEGG pathway analysis, the Wnt/ $\beta$-catenin signaling pathway was observed to be highly enriched among the downstream signaling pathways of CCEPR. In order to confirm whether CCEPR exerted a biological role in the $\mathrm{Wnt} / \beta$-catenin signaling pathway, western blotting revealed that the knockdown of CCEPR significantly decreased the expression levels of protein involved in the $\mathrm{Wnt} / \beta$-catenin signaling pathway, including cyclin D1, $\beta$-catenin, Myc and MMP-7. Thus, it was suggested that CCEPR may contribute to the progression of $\mathrm{OC}$ via regulating the $\mathrm{Wnt} / \beta$-catenin signaling pathway. 
A

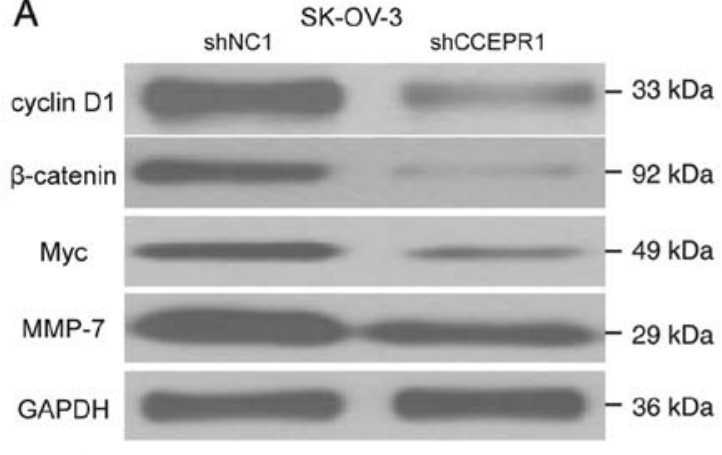

C

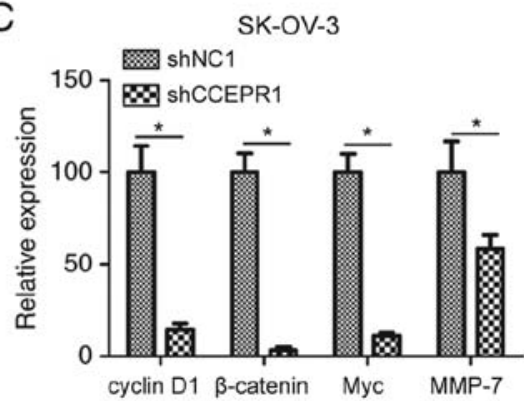

B

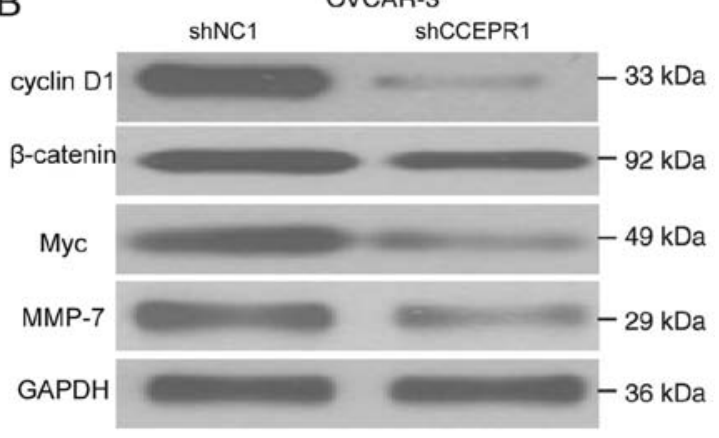

D

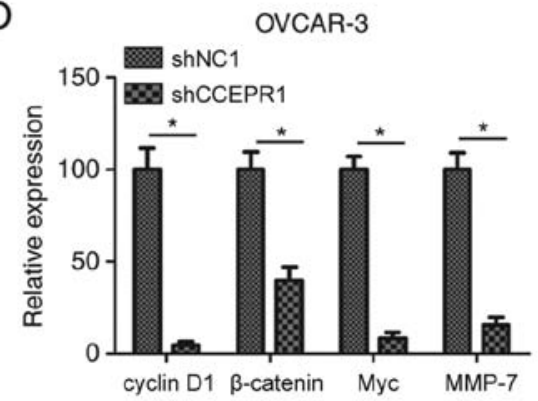

Figure 5. Wnt/ $\beta$-catenin signaling pathway is associated with the function of CCEPR in ovarian cancer cells. Expression levels of proteins involved in the Wnt/ $\beta$-catenin signaling pathway (cyclin D1, $\beta$-catenin, Myc and MMP-7) were analyzed using western blotting in (A) SK-OV-3 and (B) OVCAR-3 cells transfected with shCCEPR1 or shNC1. Cyclin D1, $\beta$-catenin, Myc and MMP-7 expression levels were significantly decreased in (C) SK-OV-3 and (D) OVCAR-3 cells transfected with shCCEPR1. Data are presented as the mean $\pm \mathrm{SD}$ of $\geq 3$ individual experiments. " $\mathrm{P}<0.05$. CCEPR, cervical carcinoma expressed PCNA regulatory lncRNA; MMP-7, matrix metallopeptidase 7; NC, negative control; sh, short hairpin RNA.

However, one limitation of the study was that only 70 cases of OC were analyzed; hence, in vivo experiments and further investigations are required in the future to explore the oncogenic mechanism of CCEPR in OC.

In conclusion, to the best of our knowledge, the present study was the first to identify that high CCEPR expression may be related to unfavorable clinicopathological behaviors and may predict a worse prognosis in patients with OC. In addition, the data suggested that CCEPR may contribute to the progression of OC through regulating the Wnt/ $\beta$-catenin signaling pathway. These findings suggested a novel direction for the potential therapeutic value of CCEPR in OC treatment in the future.

\section{Acknowledgements}

Not applicable.

\section{Funding}

No funding was received.

\section{Availability of data and materials}

The datasets used and/or analyzed during the current study are available from the corresponding author on reasonable request.

\section{Authors' contributions}

$\mathrm{ZC}$ and $\mathrm{YZ}$ designed the study, performed the experiments, analyzed the data and wrote the manuscript. XF, YL and QF performed the experiments and analyzed the data. All authors read and approved the final manuscript.

\section{Ethics approval and consent to participate}

The study was approved by the Institutional Review Board of Tianjin Central Hospital of Gynecology and Obstetrics (Tianjin, China). Written informed consent was obtained from all patients.

\section{Patient consent for publication}

Patients provided consent for publication.

\section{Competing interests}

The authors declare that they have no competing interests.

\section{References}

1. Lheureux S, Gourley C, Vergote I and Oza AM: Epithelial ovarian cancer. Lancet 393: 1240-1253, 2019.

2. Jayson GC, Kohn EC, Kitchener HC and Ledermann JA: Ovarian cancer. Lancet 384: 1376-1388, 2014.

3. Cortez AJ, Tudrej P, Kujawa KA and Lisowska KM: Advances in ovarian cancer therapy. Cancer Chemother Pharmacol 81: 17-38, 2018.

4. Naumann RW, Coleman RL, Brown J and Moore KN: Phase III trials in ovarian cancer: The evolving landscape of front line therapy. Gynecol Oncol 153: 436-444, 2019.

5. Paik ES, Shim M, Choi HJ, Lee YY, Kim TJ, Lee JW, Kim BG, Bae DS and Choi CH: Impact of lymphadenectomy on survival after recurrence in patients with advanced ovarian cancer without suspected lymph node metastasis. Gynecol Oncol 143: 252-257, 2016.

6. Worzfeld T, Pogge von Strandmann E, Huber M, Adhikary T, Wagner U, Reinartz $S$ and Müller R: The unique molecular and cellular microenvironment of ovarian cancer. Front Oncol 7: 24, 2017. 
7. Takeda T, Banno K, Okawa R, Yanokura M, Iijima M, Irie-Kunitomi H, Nakamura K, Iida M, Adachi M, Umene K, et al: ARID1A gene mutation in ovarian and endometrial cancers (Review). Oncol Rep 35: 607-613, 2016.

8. Wang KC and Chang HY: Molecular mechanisms of long noncoding RNAs. Mol Cell 43: 904-914, 2011.

9. Mercer TR, Dinger ME and Mattick JS: Long non-coding RNAs: Insights into functions. Nat Rev Genet 10: 155-159, 2009.

10. Spizzo R, Almeida MI, Colombatti A and Calin GA: Long non-coding RNAs and cancer: A new frontier of translational research? Oncogene 31: 4577-4587, 2012.

11. Fatica A and Bozzoni I: Long non-coding RNAs: New players in cell differentiation and development. Nat Rev Genet 15: 7-21, 2014

12. Expósito-Villén A, E Aránega A and Franco D: Functional role of non-coding RNAs during epithelial-to-mesenchymal transition. Noncoding RNA 4: E14, 2018.

13. Schmitt AM and Chang HY: Long noncoding RNAs in cancer pathways. Cancer Cell 29: 452-463, 2016.

14. Liu X, Wen J, Wang $\mathrm{H}$ and Wang Y: Long non-coding RNA LINC00460 promotes epithelial ovarian cancer progression by regulating microRNA-338-3p. Biomed Pharmacother 108: 1022-1028, 2018

15. Li H, Liu C, Lu Z, Chen L, Wang J, Li Y and Ma H: Upregulation of the long non-coding RNA SPRY4-IT1 indicates a poor prognosis and promotes tumorigenesis in ovarian cancer. Biomed Pharmacother 88: 529-534, 2017.

16. Luo J and Liu Z: Long non-coding RNA TTN-AS1 promotes the progression of lung adenocarcinoma by regulating PTEN/PI3K/AKT signaling pathway. Biochem Biophys Res Commun 514: 140-147, 2019.

17. Zhan Y, Li Y, Guan B, Chen X, Chen Z, He A, He S, Gong Y, Peng D, Liu Y, et al: Increased expression of long non-coding RNA CCEPR is associated with poor prognosis and promotes tumorigenesis in urothelial bladder carcinoma. Oncotarget 8 : 44326-44334, 2017

18. Yang M, Zhai X, Xia B, Wang Y and Lou G: Long noncoding RNA CCHE1 promotes cervical cancer cell proliferation via upregulating PCNA. Tumour Biol 36: 7615-7622, 2015.

19. Schmittgen TD and Livak KJ: Analyzing real-time PCR data by the comparative C(T) method. Nat Protoc 3: 1101-1108, 2008.

20. Zhan L, Li J and Wei B: Long non-coding RNAs in ovarian cancer. J Exp Clin Cancer Res 37: 120, 2018.

21. Zhong Y, Gao D, He S, Shuai C and Peng S: Dysregulated expression of long noncoding RNAs in ovarian cancer. Int J Gynecol Cancer 26: 1564-1570, 2016.
22. Wang X, Yang B, She Y and Ye Y: The lncRNA TP73-AS1 promotes ovarian cancer cell proliferation and metastasis via modulation of MMP2 and MMP9. J Cell Biochem 119: 7790-7799, 2018

23. Ma N, Li S, Zhang Q, Wang H, Qin H and Wang S: Long non-coding RNA GAS5 inhibits ovarian cancer cell proliferation via the control of microRNA-21 and SPRY2 expression. Exp Ther Med 16: 73-82, 2018.

24. Liu Y, Wang Y, Fu X and Lu Z: Long non-coding RNA NEAT1 promoted ovarian cancer cells' metastasis through regulation of miR-382-3p/ROCK1 axial. Cancer Sci 109: 2188-2198, 2018.

25. Liu Y, Wang Y, Yao D and Cui D: LncSOX4 serves an oncogenic role in the tumorigenesis of epithelial ovarian cancer by promoting cell proliferation and inhibiting apoptosis. Mol Med Rep 17: 8282-8288, 2018

26. Ota T, Suzuki Y, Nishikawa T, Otsuki T, Sugiyama T, Irie R, Wakamatsu A, Hayashi K, Sato H, Nagai K, et al: Complete sequencing and characterization of 21,243 full-length human cDNAs. Nat Genet 36: 40-45, 2004.

27. Hu XY, Hou PF, Li TT, Quan HY, Li ML, Lin T, Liu JJ, Bai J and Zheng JN: The roles of Wnt/ $\beta$-catenin signaling pathway related lncRNAs in cancer. Int J Biol Sci 14: 2003-2011, 2018.

28. Cheng X, Xu X, Chen D, Zhao F and Wang W: Therapeutic potential of targeting the Wnt/ $\beta$-catenin signaling pathway in colorectal cancer. Biomed Pharmacother 110: 473-481, 2019.

29. Arend RC, Londono-Joshi AI, Straughn JM Jr and Buchsbaum DJ: The Wnt $/ \beta$-catenin pathway in ovarian cancer: A review. Gynecol Oncol 131: 772-779, 2013

30. Huang L, Jin Y, Feng S, Zou Y, Xu S, Qiu S, Li L and Zheng J: Role of Wnt/ $\beta$-catenin, Wnt/c-Jun N-terminal kinase and Wnt $/ \mathrm{Ca}^{2+}$ pathways in cisplatin-induced chemoresistance in ovarian cancer. Exp Ther Med 12: 3851-3858, 2016.

31. Mei Y, Liu YB, Cao S, Tian ZW and Zhou HH: RIF1 promotes tumor growth and cancer stem cell-like traits in NSCLC by protein phosphatase 1 -mediated activation of $\mathrm{Wnt} / \beta$-catenin signaling. Cell Death Dis 9: 942, 2018.

32. Zeng S, Seifert AM, Zhang JQ, Cavnar MJ, Kim TS, Balachandran VP, Santamaria-Barria JA, Cohen NA, Beckman MJ, Medina BD, et al: Wnt $/ \beta$-catenin signaling contributes to tumor malignancy and is targetable in gastrointestinal stromal tumor. Mol Cancer Ther 16: 1954-1966, 2017. 